\title{
Lactancia materna y crecimiento pondoestatural durante el primer año de vida
}

\author{
1..U. Sonia Juimovich P. ${ }^{1} ;$ I. L. Cecilia Campos $\mathrm{S}{ }^{1}$; \\ Dra. Mfaría Isabel Hlodgson B. ${ }^{2}$ : Prot. Ilse M. López B ${ }^{3}$
}

\section{Growth in breastfed infants.}

Body growth during the first year of life was studied in 163 infants breastfed for at least six months, that were under health control at one Pediatric Health Center. The results were compared with N.C.H.S. standars: boys and Eirls' wejghts approximated tothe 75 th percentile during the first six months and to the 50 th purcentile at the second semester of life. In stature, girls grew tlirough 50 th percentile atong the first twelve montlis, but boys were between 25 th and 50 th percentile from the fourth month on. Thi weight gain in each of the intervals studied showed some differences with the standards used as reference in Chile but weights at the end of the first year are similar.

(Key words: Growth, breastfed infants).

La práctica de la lactancia ha experimentado una gran variabilidad a través đe los años, refiriéndose sólo al presente sig̨lo, se puede observar que en las dos primeras décadas la mayoría de las madres alimentaban al pecho a sus hijos, actitud que era fomentada por el equipo de salud. $A$ partir de la década del 40 se comenzó a observar un descenso sostenido de la lactancia materna que lleqú à un nivel mínimo en los años 70 . Este fenómeno fue producido por diferentes factores correspondiendo parte de la responsabilidad a] equipo de salud. 1,2

En la actualidad no hay consenso respecto al período durante el cual el niño puede ser alimentado al pecho en forma exclusiva y son escasos los estudios que entregan información en relación al aporte nutritivo de la lactancia materna en los distintos períodos del crecimiento ${ }^{3}$.

$\mathrm{La}$ introducción prematura de alimentación artificial implica un riesgo nutricional en los paises desarrollados y en vías de desarrollo, aunque sus consecuencias son diferentes. En los primeros, la introducción precoz de alimentación artificial favorece el sobrepeso y la obesidad ${ }^{4}$. En

1. Depto. Jinfermeria Pediátrica. liscuela de Enfermería. Pontifícia Univirsidad Católica de Chile.

2. Departamento de Pediatria. Liscuela de Mecicinil. Portlificia Universidad Catolica da Chile.

3. Fiscuela de Sulud Públiça. Facultad de Medicina. Universidad de Cluile. los páises en vías de desarrollo el riesgo más conocido es el de la desnutrición como consecucncia de mayor frecuencia de infecciones, djareas y prácticas de alimentación inadecuadas 5,6 , no siendo de spreciable el riesgo de sobrepeso.

Diversos estudios demuestran que el niño alimentado al pecho crece bien: a pesar de que los estándares con que se evalúa el crecimiento pondoestatural fueron elaborados en base de poblaciones de niños con introducción precol de sólidos y de fórmulas lácteas ${ }^{3.7 .9}$.

El objetivo del presente estudio fue describir el of recimiento pondoestatural durante el pritner año de vida de un grupo de niños alimentados con lactancia materna exclusiva durante seis me. ses o mas, y compararlo con los cstándares de National Center of Health Statistics" (N.C.H.S.) de EE.UU. de N.A.

\section{MATERIAL Y MFTODO}

La población en estudio estuvo constituida por 163 niños nacidos en el llospital ('linico de la Pontificia Universidad Católica de Chile, en el período comprendido entrt el 10 de marzo y el 31 de septiembre de 1982, que cumplías con los siguientes requisitos: peso đe nacioniento de 2.500 y 0 más, ausencia de afecciones jeonatales que determinaran hospitalización. lactancia materna exclusiva por un mínimo de 6 meses, asistencia a control periodico en el Centro de Diagnóstico de la (nuiversidad Católiea (CEDlUC) por un período mayor de $6 \mathrm{meses}$.

Se consideró como lactancia materna exclusiva la 
alimentación láctea constituida sólo por leche matema, Los 163 niños que cumplieron con este requisito correspondian aproximadamente id $50 \%$ de los lactentes asjstertes a control de salud en este periodo.

La información necesaria para este estudio fue recolectada de los registros de control existentes en la historia clinica, de la gue se anslizaron: sexo, pego $y$ talla de naciriento; numero de order del nuño. pew y taila a las edades $1,2,3,4,5,6,8,10$ y 12 meses: edad de introducción de alimentación no láctea, duración de la lactancia, coad materna

El peso tue medido con balanza $\mathrm{Sece}^{(2)}$ pata lastantes, con precisión de $10 \mathrm{~g}$ y la talla en alutropómetso de Inadera graduado con precisión de 0.5 cm. Para los efectos del segumiento del crecinichto pondoestatural se considcraron los datos de peso y talla de los niños que concurricron a control a la edad respectiva \pm 3 días en los primeros tres meses $y \pm 5$ diar para los meses restantes. Por esta rázón el total de niños considerados en cada idad es diferente.

Fi recuento y codificación de los datos $*$ realizó manualmente y cl thálisis estadistico, por compulación, obteniéndose la mediana. cl promedio y la desvjación estándard del grupo to ial y por wexo.

Se confeccionaton curvas de crecimicuto utilizando el promedio de peso $\mathrm{y}$ ialla de cada ила de las edades estudiadas por el total de riños \pm uma desviación estándar.

Se realizó la comparación ın las ourvas del Centro Nacional de Fstad isticus de Salud de E.U.A. (N.C.H.S.) utilizando la mediana $(p \$ 0)$ en forma separada para cada sexo.

\section{RESULTADOS}

Se estudiaron 163 thiños de los cuales 86 $(52,8 \%)$ eran hombres. El promedio de peso de nacimiento fue de $3.454 \pm 408 \mathrm{~g}(3.379$ para las mujeres y $3.522 \mathrm{~g}$ para los hombres) y el promedio de talla $50 \pm 1.9 \mathrm{~cm}(49.4$ para las mujeres y $50,3 \mathrm{~cm}$ para los hombres).

Cincuenta y seis por ciento de los niños eran primeros hijos y $22,6 \%$ segundos, situación similar a ta encontrada en otros estudius de esta población ${ }^{11}$.

La leche materna constituyó el único alimento lácteo en promedio hasta los 7,6 meses (DE \pm 1,71 . La edad de incorporación de fruta y de comida (pure de verduras, carnes y aceite), osciló cntre los tres meses y los ocho meses coincidiendo la mediana $y$ el promedio en los cinco meses para ambos alimentos, lo cual concuerda con las pautas de alimentación recomendadas en CEDIUC durante el período estudiado. Catbe destacar que la incorporación de estos alimentos a los tres meses se dio sólo en dos casos. El total de niños recibió suplemento vitamínico A-C-D. desde los quince dias de vida hasta los seis meses.

La edad materna en $35 \%$ estaba entre 25 y 29 años, en $29 \%$ entre 20 y 24 años y sólo $2 \%$ eran menores de 20 años.

En las Tablas 1 y 2 se describen la evolución pondoestatural del total de los niños estưđiados,
Tabla 1

Peso y talla para la edad de la población estudiada de $0-12$ meses

$(\mathrm{x} \pm 1 \mathrm{Ol})$

\begin{tabular}{|c|c|c|c|}
\hline $\begin{array}{l}\text { Edad } \\
\text { (meses) }\end{array}$ & $\mathrm{n}$ & $\begin{array}{c}P e_{n} \\
(y)\end{array}$ & $\begin{array}{l}\text { Talla } \\
(\mathrm{cm})\end{array}$ \\
\hline Nac. & 163 & $3.454 \pm 408,4$ & $49,9 \pm 1,89$ \\
\hline 2 & 13 & $4.360 \pm 499,3$ & $53.9 \pm 1,78$ \\
\hline 2 & 130 & $5.451 \pm 581,5$ & $57.3 \pm 1.93$ \\
\hline 3 & 128 & $6,271 \pm 628,8$ & $60,2 \pm 2.04$ \\
\hline 4 & 122 & $6.932 \pm 789,4$ & $62,5 \pm 2,29$ \\
\hline 5 & 155 & $?, 450 \pm 78,12$ & $64,8 \pm 2,36$ \\
\hline 6 & 104 & $7.872 \pm 846.9$ & $66,2 \pm 2.20$ \\
\hline 8 & 82 & $8.626 \pm 896,2$ & $69,4 \pm 2,24$ \\
\hline 10 & 48 & $9.222 \pm 961,0$ & $72,1 \pm 2,08$ \\
\hline 12 & 52 & $9.949 \pm 889,3$ & $74,5 \pm 2,18$ \\
\hline
\end{tabular}

Tabla 2

Incremento de peso observado en la población estudiada entre 0 y 12 meses de vida

$$
\text { (s } \pm \text { D.E.) }
$$

\begin{tabular}{|c|c|}
\hline $\begin{array}{c}\text { Intervalo } \\
\text { tadad (mests) }\end{array}$ & $\begin{array}{c}\text { Incremento } \\
\text { (g) }\end{array}$ \\
\hline $0-1$ & $918,3 \pm 332,9$ \\
\hline $1-2$ & $1.089 .7 \pm 250,9$ \\
\hline $2-3$ & $798,9 \pm 207,1$ \\
\hline $3-4$ & $646,9 \pm 218,5$ \\
\hline $4-5$ & $572,4 \pm 187,2$ \\
\hline 56 & $441,2 \pm 216,6$ \\
\hline $6-8$ & $774,6 \pm 338,9$ \\
\hline $8-10$ & $712,9 \pm 263,1$ \\
\hline $10-12$ & $668,2 \pm 307,6$ \\
\hline
\end{tabular}

* El litnile supcrior de cada intervalo corresponde al día antes del cumple mes

expresados en promedio de peso y talla \pm 1 DE. En ellas se confirma un crecimjento acelerado durante los primeros tres meses. y un ritmo algo menor en los meses siguientes.

La Tabla 2 muestra Ios incremertos mensuales de peso observados durante el primer año de vida. Destaca un incremento superior al recomendado dutante el primer trimestre, y algo inferior en el segundo y tercero. El incremento total durante cl primer año alcanza a $6.620 \mathrm{~g}$, siendo lo exigido según normas, $6.400 \mathrm{~g}^{12}$.

LaTabla 3 muestra las medianas de peso y talla para ambos sexos por separado, los que resultan comparables con los del estándar del N.C.H.S.:

La relación peso/edad de las mujeres evolucionó por el percentil 75 hasta los seis meses de edad, cambiando al p50 al finalizar el primer año de vida. El peso de los hombre evolucionó entre el percentil 50 y 75 hasta los seis meses, 
Tabla 3

Mediana (p 50 ) de peso y talla obscrvadas según cdad y sexo

\begin{tabular}{|c|c|c|c|c|c|c|}
\hline \multirow{2}{*}{$\frac{\text { Ldad }}{\text { (MS) }}$} & \multicolumn{3}{|c|}{ Hombres } & \multicolumn{3}{|c|}{ Mujeres } \\
\hline & n & $\begin{array}{l}\text { Peso } \\
\text { (g) }\end{array}$ & $\begin{array}{l}\text { Talla } \\
\text { (cm1) }\end{array}$ & $n$ & $\begin{array}{c}\text { Peso } \\
\text { (g) }\end{array}$ & $\begin{array}{l}\text { T:tla } \\
(\mathrm{cm})\end{array}$ \\
\hline 0 & 86 & 3.535 & 51,0 & 77 & 3.370 & 49,0 \\
\hline 1 & 69 & 4.500 & 55,0 & 64 & 4,235 & 53,0 \\
\hline 2 & 69 & 5.600 & 58,0 & 61 & 5.200 & 56,5 \\
\hline 3 & 73 & 6.400 & 61,0 & 55 & 6.000 & 59,0 \\
\hline 4 & 63 & 7.150 & 63,5 & 59 & 6.570 & 62,0 \\
\hline 5 & 6.5 & 7.500 & 65,0 & 50 & 7.125 & 63,8 \\
\hline 6 & 51 & 7.700 & 67,0 & 53 & 7,680 & 65,5 \\
\hline 8 & 43 & 8.550 & 70,0 & 39 & 8.480 & 68,8 \\
\hline 10 & 24 & 9.365 & 72,0 & 24 & 8.750 & 72,0 \\
\hline 12 & 24 & 10.145 & 75,5 & 28 & 9.600 & 73,0 \\
\hline
\end{tabular}

manteniéndose luego levemente bajo el percentil 50 hasta los doce meses.

En las relaciones tallafedad las mujeres crecieron por el percentil 50 a lo largo de todo el primer año. La talla de los hombres evolucionó por el percentil 50 hasta los 4 meses, manteniendose durante el resto del año entre el percentil 25 y 50 .

La relación peso/talla para ambos sexos cursó entre el percentil 50 y 75 del cstándas del N.C.H.S. durente los doce primcros meses de vidi.

\section{DISCUSION}

Diversos estudios en países desarrollados muestran un adecuado crecimiento pondoestatural en niños alimentados con lactancia materna exclusivid hasta los seis meses. $\Lambda$ respecto hay pocas publicaciones de relevancia en poblaciones de países en desarrollo: los datos disponibles sugieren un buen incremento ponderal los primeros tres meses y disminución del mismo chtre los tres y seis meses 5

El análisis de la evolución del peso y la talla de este estudio muestra que es posible un crecimicnto comparable con estandares internacionales ${ }^{10}$, en niños que reciben lactancia materna hasta el sexto mes de vida. Estos resultados concuerdan con los comunicados por otros auto. res nacionales y extranjeros $3,7,8,9$

La Organización Mundial de la Salud (OMS) recomienda el uso de los estándares del N.C.H.S. como referencia internacional para la compara. ción del crecimiento de diferentes poblaciones ${ }^{13}$. En nuestro pais ha sido discutido el uso de dichos patrones, optándose por las tablas de Sempé, con menores exigencias de incrementos de peso y talla. No existen patrones disponibles del crecimiento pondeestatural para nitios con alimentación materna exclusiva durante los primeros seis meses. Los resultados obtenidos en este estudio permiten concluit que los estándares del N.C.H.S. son aplicables al crecimiento pondoestatural de esta población, aún cuande en la construccion de dichos patrones sc incluyeron alrededor de $50 \%$ de niños que no recibieron lactancia materna, $y$ en los demás una proporción importante recibió precozmente suplementos no lácteos. Hay estudios que nuestran que el crecimiento de grupos de población infantil de nivel sociocionomico alto de países en desarrolio es similar al de niños en países desarrollados, lo que se debe a una nutrición adecuada, mejores condiciones ambientales y menor proporción de infecciones ${ }^{5}$. Al respecto es importante considerar que la población materno-infantil controlada en CEDIUC pertenece a un estrato socioeconomico medro según la clasificación de Graffar ${ }^{14}$; un alto porcentaje de las madres tienen enseñanza media completa y alrededor de $25 \%$ tienen ensefianza superior completa ${ }^{11}$; además $35 \%$ tienen entre 25 y 29 afios de edad, características que favorecian el crecimiento y desarrollo de los niños.

En general hay acuerdo en que la lactancia exclusiva es lis forma recomendable para alimentar niños sanos mientras se mantenga un ritmo de crecimiento satisfactorio. La edad en la que se requieren dlimentos adictonales depende de muchos factores interrclacionados, incluyendo la salud de la madre, su estado nutricional, la calidad y cantidad de leche producida, el peso de nacimiento del niño, la edad gestacional, y su estado de salud. Por esta tazón la edad de término de la lactancia materna exclusiva yaría en 
distintas poblaciones y en diferentes individuos, de una misma población ${ }^{14,15}$; en los controles de salud conviene hacer énfasis en la educación destinada al fomento de la lactancia natural, introduciendose relleno sólo después de la confir. mación de ut mal incremento ponderal.

Simes demostrô buenos depósitos de hierro hasta los seis meses en ninyos alimentados con lactanctia materna exclusivit ${ }^{16}$. La absorción de hierro de la leche materna disminuye cuando se administran sólidos en forma simultánea ${ }^{3}$. por lo que es recomendable retrasar la ingesta de sólidos hasta los seis meses en niños que reciben lactancia materna y presentan buen incremento ponduestatural. Dada la introducción de sólidos a los cinco meses de edad en los niños de este estudio la leche materna constituyó prásticamente el único apurte alimentario hasta ese momento, y alsededor de $80 \%$ del mismo en e] sexto mes. Aún cuando en este estudio no se consideraron exámenes hematológicos para evaluar estado nutricional de hierro. una publicación nacional reciente mostró valores hematológicos adecuados a los seis meses de edad en niños con bactancia materna exclusiva ${ }^{7}$.

Fis probable que las recomendiciones de nu. lrientes $^{18,19}$, sobrestimen las necesidades de muchos individuos de una población. ya que comsideran la variabilidad de requerimientos entre individuos, y sc establecen en un nivel de dos desvidciones estándar por sobre el promedio de requeri. mientus ${ }^{15}$. Un estudio reciente ${ }^{3}$, in el cual se analizó la leche materna que estaba recibiendo un grupo de niños do 0 a 4 meses, mostrú adecuado crecimicntu con ingestión sustancialmente menor de mutrientes que las reconendaciones, lo que refuerza la idea que los requerimientos del niño durante el primer semestre de vida pueden ser cubiertos por la leche materna. Dado que en algunos casos esto no ocurre, es importante mantener ans vigilancia perićdica del progreso pondivestatural.

FIl incremento mensual de peso se ha considerudo como un indicador más sensible del crecimiento ponderal, y a la vez independiente del peso de nacimiento. Sus limitaciones residen en la falta de referencias al aumento mensual. El N.C.H.S. se basa en medidas al nacer, uno, 3 y 6 meses de edad infiriendo los valores de los otros meses intermedios ${ }^{5}$. Los resultados de este estudio muestran una distribución de incrementos de peso diferente a la que se está exigiendo actualmepte en nucstro país en las distintas edades, pero, il final del año, se alcanza una cifra similar o incluso algo superior. Este aspecto cobra importancia si se piensa que este parámetro es utilizado en el país para decidir la introducción de lactancia artificial y clasificar niños con riesgo de desnutrición.

\section{RESUMEN}

El crecimiento pondoestatural, durante el primer año de vida fue estudiado en 163 niños alimentados con leche materna por seís meses 0 más $(\bar{x} 7.6 \mathrm{~m})$, en quienes los alimentos sólidos so introdujcron entre los 3 y 8 meses de edad (medianta 5 meses). Los resultados obtenidos fueron comparados con los estándares del N.C.H.S. Pata ambos sexos el peso evolucionó cerca del percentil 75 en los primeros seis meses $y$ se acerca del percentil 50 durante el segundo semestre. En la talla de mujeres crecieron por el percentil 50 todo el año, y los hombre a partir del $4^{0}$ mes se mantuvieron entre el percentil $25 \mathrm{y}$ 50. La distribución de los incrementos ponderales observados en cadid uno de los intervalos de control. fuc diferente a la exigida en el control periódico de salud de acuerdo a las normats actualmente en uso en nuestro medio, pero los resultados finales en términos de peso y talla al año de edad fueron semejantes a los del estándar empleado.

\section{REFERENCIAS}

1. Vora L., Anderson, K., Cruz, A., Jeldrez, V., Jimenez, J., Patri, $A_{\text {.: Influencia de la actitud }}$ médica en la duración de lis lastancia natural. Pediattía (Santiago), 18:67, 1975.

2 Mardones, F.: Marco histósico para cl análisis de las causas del descenso en la prática de la lactancia ma terna. Rev Chil Pediutr 53: 607, 1982.

3. Butte, $N$, Garza, C., Smith, Smith, E., Nichols, B.: II uman in take and growth in exclusjucly breast-fed infants. J Pedjatr 104: 187, 1984.

4. Evars, II., Glass, L.: Bresst Feeding: Adrantages and Potencial Problems. Pediatr Ann B: $110,1979$.

5. Seword, J., Serdula, M.: Inflant Fecding and infant growth. Pediatrics 74: 728, 1984.

6. Lopez, 1., Cabiol, C., Arcuch. S., Rivera, E., Vargas, $S$. San Miguel A.: Lactancia materna y morbilidad por diarrea y desnutricion en el primer año de vida. Rev Chil Pediatr S3: 162, 1982

7. Juez, G., Diaz, S., Peralta, O., Croxatto, H., Casado. $M, E_{.}$. Salvatierra, A., Durán, E., Fernändez, $M_{.:}$ Luclancia materna exclusiva: Crecimiento del lactantc en un grupo seleccionado de nitios chilenos. Kev C.ill Pediatr 55: 225, 1984.

8. López. I. Cabiol, C., Arcuch, S, Rivera, E., Vargas. $S$ : Lactancia materna y aumento de peso en el primer año de vida. Rev Chil Pediatr 5 l: 473. 1.980.

9. Ahn, Ch., Mac Lean W: Growt of the exclusively brisast-led infant. Am J Clin Nutr 3. 183, 1980.

10 Hamill, P., Drizd. T., Johnson, C., Reed, R., Roche, $A$. Moore, W: Physical growth: National Center for Health Statistics percentiles. Am J Clin Nutr 32: $607,1979$.

11. Campos, C., Fereda, C., López, I: Las mujeres que spperan un hijo: conocimicntos y actitudes de crianza en algunos aspectos preventivos de Salud. Tesis de grado. Escuela de Salud Pública, Universidad de Chile, 1985. 
12. Ministerio de Salud: Nomas de Pediatria: Accioncs de Fomento y Protección. Chile, 1976.

13. Hodgson, M.I.: Evalueión del estado nutricional del lactante. Boletín de la Escutla de Medicina, Universidad Católica (Chile) 16:10, 1986 ,

14. Valenzuelo, J.: Empleo de un nuevo método do clasificación social. Cuadcrnos Médico Socieales (Chile) 17: 14. 1976 .

15. Anderson. $G$ Human milk fecding. Pediatr Clin North Am 32: 335, 1985.

16. Simes, M., Salmenpera I... Perhaentrupa, J.: Fxclu- sive breast-feeding for 9 months: Risk of iron deficiency. J Pediatr l04: 196, 1984. .

17. Oski, F, Landaw. S: Inhibition of iron absortion from human milk by baby food. $\Lambda$ m J Child 134: 459,1980 .

18. Fomon, S.J., Filer. L.J., Anderson, T.A., Ziegler E.E.: Recomendations for feeding normal infants. Pediatrics 63: 52, 1979.

19. FAO/WHO/UNU: J oint Expert Consultation: linergy and Protein Requirements. WYO Technical Report Series, 1984. 